\title{
Construction of Xinjiang metabolic syndrome risk prediction model based on interpretable models
}

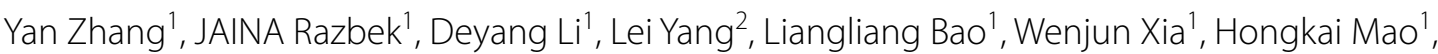 \\ Mayisha Daken ${ }^{1}$, Xiaoxu Zhang ${ }^{1}$ and Mingqin Cao ${ }^{1 *}$
}

\begin{abstract}
Background: We aimed to construct simple and practical metabolic syndrome (MetS) risk prediction models based on the data of inhabitants of Urumqi and to provide a methodological reference for the prevention and control of MetS.

Methods: This is a cross-sectional study conducted in the Xinjiang Uygur Autonomous Region of China. We collected data from inhabitants of Urumqi from 2018 to 2019, including demographic characteristics, anthropometric indicators, living habits and family history. Resampling technology was used to preprocess the data imbalance problems, and then MetS risk prediction models were constructed based on logistic regression (LR) and decision tree (DT). In addition, nomograms and tree diagrams of DT were used to explain and visualize the model.
\end{abstract}

Results: Of the 25,542 participants included in the study, 3,267 (12.8\%) were diagnosed with MetS, and 22,275 (87.2\%) were diagnosed with non-MetS. Both the LR and DT models based on the random undersampling dataset had good AUROC values ( 0.846 and 0.913 , respectively). The accuracy, sensitivity, specificity, and AUROC values of the DT model were higher than those of the LR model. Based on a random undersampling dataset, the LR model showed that exercises such as walking $(O R=0.769)$ and running $(O R=0.736)$ were protective factors against Met $S$. Age $60 \sim 74$ years $(\mathrm{OR}=1.388)$, previous diabetes $(\mathrm{OR}=8.902)$, previous hypertension $(\mathrm{OR}=2.830)$, fatty liver $(\mathrm{OR}=3.306)$, smoking $(\mathrm{OR}=1.541)$, high systolic blood pressure $(\mathrm{OR}=1.044)$, and high diastolic blood pressure $(\mathrm{OR}=1.072)$ were risk factors for MetS; the DT model had 7 depth layers and 18 leaves, with BMI as the root node of the DT being the most important factor affecting MetS, and the other variables in descending order of importance: SBP, previous diabetes, previous hypertension, DBP, fatty liver, smoking, and exercise.

Conclusions: Both DT and LR MetS risk prediction models have good prediction performance and their respective characteristics. Combining these two methods to construct an interpretable risk prediction model of MetS can provide methodological references for the prevention and control of MetS.

Keywords: Metabolic syndrome, Interpretable model, Prediction, Risk factors

\footnotetext{
*Correspondence: 573596229@qq.com

${ }^{1}$ Department of Epidemiology and Health Statistics, College of Public

Health, Xinjiang Medical University, Urumqi, Xinjiang, China

Full list of author information is available at the end of the article
}

\section{Introduction}

Metabolic syndrome (MetS) is a type of metabolic disorder characterized by central obesity, hypertension, hyperglycaemia and dyslipidaemia [1]. It is worth noting that the prevalence of MetS is on the rise due to rapid economic growth, an ageing population, lifestyle changes, 
and obesity. The prevalence of MetS in the world is approximately $20-25 \%$ [2], and the prevalence of MetS in China is approximately 13.6-46.3\% [3-5]. Studies have shown that MetS is associated with an increased risk of diabetes, cardiovascular disease, cancer and even death [6]. MetS is an increasingly serious major public health problem and clinical challenge worldwide [7]. Therefore, appropriate prevention and control strategies must be adopted to reduce the incidence of MetS. Health checkups are the first stage of disease prevention, and data mining of medical checkup information can help identify people at high risk of MetS at an early stage, thus moving the timing of disease prevention and control forwards. The construction of MetS risk prediction models based on physical examination data is important for the prevention and control of MetS.

Disease risk prediction models predict the probability of disease occurrence by estimating the extent to which changes in one or more influencing factors affect health or disease [8] and are designed to identify people at high risk for a target disease so that effective interventions can be implemented before or early in the disease process. Therefore, the establishment of a MetS risk prediction model is of great practical importance for the early detection and intervention among people at high risk of MetS. The occurrence and development of MetS is the result of a combination of factors [9], including unchangeable factors such as sex, age and genetic history of the disease [10], and modifiable factors such as lifestyle, anthropometric indicators and blood parameters [11]. Among them, modifiable risk factors refer to factors that can be changed in principle. Accurately identifying modifiable risk factors for MetS and modifying them are effective measures to prevent and control MetS.

However, there are complex interactions between risk factors that do not facilitate the identification of MetS risk factors. Machine learning is an algorithm-based data analysis technology that has powerful data analysis capabilities and can effectively deal with complex relationships between variables. Considering these factors, this research constricted a risk prediction model for MetS based on machine learning methods.

Machine learning has proven to be effective in handling a large number of predictors while generating powerful predictive models to identify individuals at risk of developing diseases [12, 13]. Considering that medical field applications are more concerned about the transparency and interpretability of the models, we used two interpretable models of machine learning algorithms, logistic regression (LR) and decision tree (DT). LR uses equations to describe the relationship between input and target variables, which makes the prediction process simple. In DT, relying on a tree structure to classify samples and thus the interpretation of the prediction is pretty straightforward, using natural visualization $[14,15]$. The classification process of DT starts from the root node, then searches from top to bottom along a branch, and finally uses the class label value of the leaf node as the class label to which the sample belongs.

In this paper, to establish a simple and practical risk prediction model for MetS, we built two interpretable models, LR and DT, based on easily available indicators such as demographic characteristics, anthropometric indicators, living habits, and family history of the subjects and then used a nomogram and tree diagram of the DT to explain and visualize the model. The interpretable MetS risk prediction model can help uncover risk factors, identify high-risk individuals, and provide methodological references for the prevention and control of MetS.

\section{Methods}

\section{Research population}

The data came from the health records database of the Dekang Cihui Medical Examination Center in Urumqi, Xinjiang Uygur Autonomous Region, China. A total of 37,457 medical examination reports were collected from January 2018 to December 2019. According to the exclusion criteria, we first deleted the data of 9199 subjects with missing diagnostic component data for MetS, then deleted 14 subjects younger than 18 years old, and finally had 25,542 as our research subjects. This study was approved by the Ethics Committee of the First Affiliated Hospital of Xinjiang Medical University, and all methods were carried out in accordance with relevant guidelines and regulations. The Ethics Committee of the First Affiliated Hospital of Xinjiang Medical University waived the need for informed consent.

\section{Data collection}

All subjects needed to fast for $8 \sim 10 \mathrm{~h}$ before the health check-up. Data were collected by staff with uniform professional training. Demographic characteristics included the age and sex of the subjects. The anthropometric indices included height, weight, heart rate and blood pressure, and standard measuring instruments were used. Height and waist circumference were accurate to $0.1 \mathrm{~cm}$, and weight was accurate to $0.1 \mathrm{~kg}$. Body mass index (BMI) was calculated as weight in kilograms divided by height in metres squared. The blood pressure of the subjects in the sitting position was measured by using a mercury sphygmomanometer according to a standardized protocol. The subjects were asked to avoid strenuous exercise and drinking caffeinated beverages within $30 \mathrm{~min}$ before the measurement and to rest for at least $5 \mathrm{~min}$ before the first measurement, with an interval of 1 to 2 min between each measurement. Blood parameters included fasting 
blood glucose (FPG), triglycerides (TG), total cholesterol (TC), high-density lipoprotein cholesterol (HDL-C), and low-density lipoprotein cholesterol (LDL-C), which were measured by an automated chemistry analyser (Beckman Coulter chemistry analyser AU5800 series, Tokyo, Japan).

Questionnaire information included diabetes, hypertension, fatty liver, family history of hypertension, family history of diabetes, family history of coronary heart disease (CHD), family history of stroke, smoking (nosmoking meant never had smoking behaviour; smoking referred to smoking in the past 30 days at the time of investigation; quit smoking meant no longer smoking in the past 30 days at the time of the survey), drinking (nodrinking meant never drinking alcohol; sometimes drinking meant drinking less than 1 time per week in the past year; often drinking meant drinking $\geq 1$ time/week in the past year; quit drinking meant no longer drinking in the past 30 days), exercise, and eating habits.

\section{Diagnostic Criteria for MetS}

Using the diagnostic criteria recommended by the Chinese Diabetes Society [16], if at least three of the following components are present, the patient is diagnosed as MetS: (1) Overweight and/or obesity: $B M I \geq 25$; (2) Hyperglycaemia: fasting blood glucose $\geq 6.1 \mathrm{mmol} / \mathrm{L}$, and/or those who have been diagnosed with diabetes and treated; (3) Hypertension: systolic/diastolic blood pressure $\geq 140 / 90 \mathrm{mmHg}$, and/or those who have been diagnosed with hypertension and treated; (4) Dyslipidaemia: $\mathrm{TG} \geq 1.7 \mathrm{mmol} / \mathrm{L}$, and $/$ or $\mathrm{HDL}-\mathrm{C}<0.9 \mathrm{mmol} / \mathrm{L}$ (male), $<1.0 \mathrm{mmol} / \mathrm{L}$ (female).

\section{Data preprocessing}

There were some missing data in the dataset, and the amount of missing data could not be ignored, so data filling was needed. Multiple imputation (MI) is a popular method to address missing data and has the characteristics of flexibility and robustness [17]. Compared with other methods, MI has certain advantages in dealing with missing data [18]. Therefore, MI was used to fill the missing data in the study to make full use of the dataset. We randomly split the dataset $(\mathrm{n}=25,542)$ into a training dataset $(70 \%, \mathrm{n}=17,879)$ and a test dataset $(30 \%$, $\mathrm{n}=7663$ ) according to a 7:3 ratio. Among the subjects in the training dataset, there were 15,599 (87.2\%) nonmetabolic syndrome (non-MetS) and 2280 (12.8\%) MetS patients. In the test dataset, there were 6,676 (87.1\%) non-MetS and 987 (12.9\%) MetS patients. The imbalance ratio of the training dataset and test dataset was 7:1. Studies have shown that in an imbalanced dataset, if a small number of classes are used as the research target, the sensitivity of building a model is usually very low [19], which is a difficult problem for researchers.
Resampling technology is a useful preprocessing step to solve imbalanced problems [20]. It can modify the imbalance distribution of the majority and minority classes at the data level before training with classifiers. For most imbalanced datasets, the application of resampling technology improved the performance of the classifier [21]. Resampling technology is mainly divided into oversampling, undersampling and hybrid sampling [22]. Two commonly used and effective methods for oversampling are random oversampling and the synthetic minority oversampling technique (SMOTE). Random oversampling adds new samples by randomly copying samples in the minority class. SMOTE analyses minority samples and artificially synthesizes new samples based on the minority samples to add to the dataset [23]. Random undersampling randomly removes samples from the majority class until both classes are equally balanced. Hybrid sampling oversamples minority samples with replacement and undersamples the majority samples without replacement to balance the class distribution. Considering that these four methods have their own characteristics, this study used random oversampling, SMOTE, random undersampling, and hybrid sampling to process the unbalanced training dataset.

\section{Risk prediction model Logistic regression}

LR is a probabilistic nonlinear regression model that can analyse the relationship between one or more factors (independent variables) and observations (dependent variables). The dependent variable can be a categorical variable or a rank variable. LR has advantages in interpreting model results and calculation costs [24]. Let $\mathrm{P}(\mathrm{y}=1 \mid \mathrm{X})$ represent the probability of an individual's onset when the exposure factor is $\mathrm{X}$, and the ratio of the probability of onset $P$ to the probability of not onset 1-P is the odds; then, $\operatorname{logit} P$ is the log of odds:

$$
\operatorname{logit} P=\ln \left(\frac{P}{1-P}\right)
$$

\section{LR model is:}

$$
\log i t P=\beta_{0}+\beta_{1} x_{1}+\beta_{2} x_{2}+\cdots+\beta_{m} x_{m}
$$

The constant term $\beta_{0}$ represents the natural logarithm of the ratio of the individual's onset and nononset probability when the exposure dose is 0 , and the regression coefficient $\beta_{j}(j=1,2, \cdots, m)$ represents the amount of change in $\operatorname{logit} P$ when the independent variable changes by one unit.

The nomogram is based on the LR model, which integrates multiple predictors and uses scaled lines drawn on the same plane at a certain scale to express the 
interrelationships among the variables in the prediction model. It transforms complex regression equations into visual graphs so that the contribution of the predictor variables to the outcome can be reflected visually and directly [25]. Therefore, this study established a nomogram to evaluate the risk of MetS in an intuitive and easy-to-understand manner.

\section{Decision tree}

DT is a nonparametric method that has many advantages, including dealing with the nonlinear relationship between variables and low computational overhead, and the model results are easy to interpret [26], so it is widely used in various fields. However, DT learning algorithms may produce models that are overly complex and/or biased if the data are imbalanced [27]. The C4.5 decision tree is the most commonly used DT algorithm, which inherits all of the advantages of the ID3 algorithm and improves on it. The C4.5 algorithm uses a top-down recursive method and uses the information gain rate as the criterion for selecting branch attributes. Assuming that the sample set is $S$, the sample attribute $A$ has $v$ possible values; that is, the sample set $S$ can be divided into $v$ subsample sets $\left\{S_{1}, S_{2} \cdots S_{v}\right\}$ through attribute A, and $\operatorname{Gain}(S, A)$ is the information gain corresponding to attribute A. The information gain rate Gain_Ratio of attribute $\mathrm{A}$ is defined as:

$$
\operatorname{SplitInfo}_{A}(S)=-\sum_{j=1}^{v} \frac{\left|S_{j}\right|}{|S|} \times \log _{2}\left(\frac{\left|S_{j}\right|}{|S|}\right)
$$

$$
\operatorname{Gain} \_\operatorname{Ratio}(A)=\operatorname{Gain}(A) / \operatorname{SplitInfo}(A)
$$

\section{Statistical analysis}

Data were preprocessed and analysed using $\mathrm{R}$ statistical software (version 3.6.0, http://www.r-project.org). Baseline characteristics of the subjects included in the study were described statistically, quantitative variables were described using the mean and standard deviation, and qualitative variables were described using cases and percentages. The baseline characteristics of subjects with MetS and non-MetS were compared based on the type and characteristics of the data, the quantitative variables were compared using independent sample t-tests, the categorical variables were compared using chi-square tests, and the ranking data were compared using the Kruskal-Wallis $\mathrm{H}$ test. The training dataset was used to train the model, and the test dataset was used to evaluate the effect of the model. The training dataset was resampled using the ROSE package, and then the LR and DT models were trained using the rms package and the party package, respectively. The Hosmer-Lemeshow goodness-of-fit test was used to evaluate the model calibration by comparing the observed and predicted probabilities. A value of $P_{\mathrm{HL}}>0.05$ indicated satisfactory calibration. The prediction performance of the model was evaluated by the area under the receiver operating characteristic curve (AUROC), sensitivity, specificity, and accuracy. Among them, the AUROC value is not affected by the data imbalance and reflects the objective [28]. Their values are between $0 \sim 1$, and the closer the value is to 1 , the better the model classification accuracy. The AUROC values and $95 \%$ CIs of the models were calculated and compared using MedCalc statistical software (version 15.6.1, https://www.medcalc.org). Two-tailed $P$ $<0.05$ was considered statistically significant.

\section{Results}

\section{Baseline characteristics}

Of the 25,542 participants included in the study, 3,267 (12.8\%) were diagnosed with MetS, and 22,275 (87.2\%) were diagnosed with non-MetS. A comparison of baseline characteristics between MetS and non-MetS patients is shown in Table 1. The MetS and non-MetS groups had statistically significant baseline characteristics, including sex, age, heart rate, previous diabetes, previous hypertension, fatty liver, smoking, drinking, hypertension family history, family history of diabetes, exercise, eating habits, BMI, SBP, and DBP $(P<0.05)$.

\section{Building risk prediction models Comparing model classification performance}

We selected statistically significant variables for LR (Model 1 Model 5) and DT (Model 6 Model 10) multivariate analysis based on five datasets: original imbalanced training dataset, random oversampling, random undersampling, hybrid sampling, and SMOTE. The comparison of the classification performance of each model on the test dataset is shown in Table 2. Compared with the original dataset, the random oversampling, random undersampling, hybrid sampling and SMOTE datasets had decreased accuracy and specificity on LR and DT but increased sensitivity and AUROC values. Both LR and DT models based on random undersampling datasets had better AUROC values. The accuracy, sensitivity, specificity, and AUROC values of the DT model were higher than those of the LR model. The ROC curves of the LR and DT models are shown in Figs. 1 and 2.

\section{Logistic regression model}

The LR model based on a random undersampling dataset was used to analyse the factors influencing MetS, as shown in Table 3. The LR model Hosmer-Lemeshow good of fit test $X^{2}=10.691, P=0.138$, showed that the model fits well. The results showed that age, previous 
Table 1 Comparison of baseline characteristics between MetS and Non-MetS ( $n(n \%) / \bar{x} \pm s)$

\begin{tabular}{|c|c|c|c|c|}
\hline Variable & Non-MetS $(n=22,275)$ & MetS $(n=3267)$ & $t / \chi^{2} / H$ & $P$ value \\
\hline \multicolumn{5}{|l|}{ Gender } \\
\hline Male & $10,897(82.7)$ & $2282(17.3)$ & 499.737 & $<0.001$ \\
\hline Female & $11,378(92.0)$ & $985(8.0)$ & & \\
\hline \multicolumn{5}{|l|}{ Age (years) } \\
\hline $18 \sim 44$ & $13,696(93.0)$ & 1023(7.0) & 1220.867 & $<0.001$ \\
\hline $45 \sim 59$ & 6193(81.9) & $1371(18.1)$ & & \\
\hline $60 \sim 74$ & 1963(72.6) & $741(27.4)$ & & \\
\hline $75 \sim 89$ & $423(76.2)$ & $132(23.8)$ & & \\
\hline Heartrate & $68.88 \pm 9.15$ & $73.30 \pm 10.87$ & -22.088 & $<0.001$ \\
\hline \multicolumn{5}{|c|}{ Previous diabetes } \\
\hline No & $21,858(89.8)$ & $2487(10.2)$ & 3088.048 & $<0.001$ \\
\hline Yes & $417(34.8)$ & $780(65.2)$ & & \\
\hline \multicolumn{5}{|c|}{ Previous hypertension } \\
\hline No & 20,792(91.0) & 2060(9.0) & 2773.785 & $<0.001$ \\
\hline Yes & $1483(55.1)$ & $1207(44.9)$ & & \\
\hline \multicolumn{5}{|l|}{ Fatty liver } \\
\hline No & $17,341(93.8)$ & $1137(6.2)$ & 2638.776 & $<0.001$ \\
\hline Yes & $4934(69.8)$ & $2130(30.2)$ & & \\
\hline \multicolumn{5}{|l|}{ Smoking } \\
\hline No & $15,035(90.1)$ & 1653(9.9) & 415.572 & $<0.001$ \\
\hline Quit & $997(75.4)$ & $325(24.6)$ & & \\
\hline Yes & $6243(82.9)$ & $1289(17.1)$ & & \\
\hline \multicolumn{5}{|l|}{ Drinking } \\
\hline No & $8642(87.9)$ & $1185(12.1)$ & 234.245 & $<0.001$ \\
\hline Quit & 164(78.5) & $45(21.5)$ & & \\
\hline Sometimes & $12,362(88.1)$ & 1664(11.9) & & \\
\hline Often & $1107(74.8)$ & $373(25.2)$ & & \\
\hline \multicolumn{5}{|c|}{ Family history of hypertension } \\
\hline No & $13,504(88.6)$ & 1745(11.4) & 61.580 & $<0.001$ \\
\hline Yes & $8771(85.2)$ & 1522(14.8) & & \\
\hline \multicolumn{5}{|c|}{ Family history of diabetes } \\
\hline No & $18,766(87.5)$ & $2676(12.5)$ & 11.547 & 0.001 \\
\hline Yes & $3509(85.6)$ & $591(14.4)$ & & \\
\hline \multicolumn{5}{|c|}{ Family history of $\mathrm{CHD}$} \\
\hline No & $20,356(87.2)$ & 2994(12.8) & 0.243 & 0.622 \\
\hline Yes & 1919(87.5) & $273(12.5)$ & & \\
\hline \multicolumn{5}{|c|}{ Family history of stroke } \\
\hline No & $22,019(87.2)$ & $3230(12.8)$ & 0.007 & 0.933 \\
\hline Yes & $256(87.4)$ & $37(12.6)$ & & \\
\hline \multicolumn{5}{|l|}{ Exercise } \\
\hline Hardly & $9272(88.3)$ & $1227(11.7)$ & 116.049 & $<0.001$ \\
\hline Walk & $9049(84.7)$ & $1631(15.3)$ & & \\
\hline Run and others & 3954(90.6) & $409(9.4)$ & & \\
\hline \multicolumn{5}{|l|}{ Eating habits } \\
\hline Light & $3065(88.3)$ & $406(11.7)$ & 29.005 & $<0.001$ \\
\hline General & $12,242(87.8)$ & $1703(12.2)$ & & \\
\hline Sweet & $274(87.3)$ & $40(12.7)$ & & \\
\hline Salty & $6390(85.9)$ & $1051(14.1)$ & & \\
\hline Meat & 304(81.9) & $67(18.1)$ & & \\
\hline
\end{tabular}


Table 1 (continued)

\begin{tabular}{|c|c|c|c|c|}
\hline Variable & Non-MetS $(n=22,275)$ & MetS $(n=3267)$ & $t / \chi^{2} / H$ & $P$ value \\
\hline \multicolumn{5}{|l|}{ BMI $\left(\mathrm{kg} / \mathrm{m}^{2}\right)$} \\
\hline$<18.5$ & 1060(100.0) & $0(0.0)$ & 4267.304 & $<0.001$ \\
\hline $18.5 \sim 23.9$ & $11,168(99.0)$ & $110(1.0)$ & & \\
\hline $24 \sim 26.9$ & 6444(84.4) & 1192(15.6) & & \\
\hline $27 \sim 29.9$ & $2690(67.8)$ & $1277(32.2)$ & & \\
\hline$\geq 30$ & $913(57.0)$ & $688(43.0)$ & & \\
\hline $\mathrm{SBP}(\mathrm{mmHg})$ & $121.49 \pm 17.80$ & $146.62 \pm 16.44$ & -80.725 & $<0.001$ \\
\hline $\mathrm{DBP}(\mathrm{mmHg})$ & $75.63 \pm 10.77$ & $90.57 \pm 10.74$ & -74.235 & $<0.001$ \\
\hline
\end{tabular}

$S B P$ systolic blood pressure; $D B P$ diastolic blood pressure

Table 2 Classification performance comparison between the DT and LR models

\begin{tabular}{|c|c|c|c|c|c|}
\hline Model & Datasets & Accuracy & Sensitivity & Specificity & AUROC $(95 \% \mathrm{Cl})$ \\
\hline \multicolumn{6}{|l|}{ LR } \\
\hline Model 1 & Original imbalanced & 0.901 & 0.418 & 0.971 & $0.694(0.684 \sim 0.704)$ \\
\hline Model 2 & Random oversampling & 0.839 & 0.854 & 0.837 & $0.846^{*}(0.837 \sim 0.854)$ \\
\hline Model 3 & Random undersampling & 0.843 & 0.851 & 0.842 & $0.846^{*}(0.838 \sim 0.854)$ \\
\hline Model 4 & Hybrid sampling & 0.839 & 0.856 & 0.837 & $0.847^{*}(0.838 \sim 0.855)$ \\
\hline Model 5 & SMOTE & 0.838 & 0.855 & 0.836 & $0.846^{*}(0.837 \sim 0.854)$ \\
\hline \multicolumn{6}{|l|}{ DT } \\
\hline Model 6 & Original imbalance & 0.915 & 0.588 & 0.962 & $0.775(0.766 \sim 0.785)$ \\
\hline Model 7 & Random oversampling & 0.874 & 0.942 & 0.864 & $0.903^{\#}(0.896 \sim 0.910)$ \\
\hline Model 8 & Random undersampling & 0.879 & 0.959 & 0.868 & $0.913^{\#}(0.907 \sim 0.919)$ \\
\hline Model 9 & Hybrid sampling & 0.873 & 0.926 & 0.866 & $0.896^{\#}(0.889 \sim 0.902)$ \\
\hline Model 10 & SMOTE & 0.851 & 0.920 & 0.841 & $0.880^{\#}(0.873 \sim 0.888)$ \\
\hline
\end{tabular}

* $P<0.05$ compared with the AUROC value of Model 1. \# $P<0.05$ compared with the AUROC value of Model 6.

diabetes, previous hypertension, fatty liver, smoking, exercise, SBP and DBP were all associated with MetS $(P<0.05)$. Among these factors, exercises such as walking $(\mathrm{OR}=0.769)$ and running $(\mathrm{OR}=0.736)$ were protective factors for MetS, but age $60 \sim 74$ years $(\mathrm{OR}=1.388)$, previous diabetes $(\mathrm{OR}=8.902)$, previous hypertension $(\mathrm{OR}=2.830)$, fatty liver $(\mathrm{OR}=3.306)$, smoking $(\mathrm{OR}=1.541)$, high systolic blood pressure $(\mathrm{OR}=1.044)$, and high diastolic blood pressure $(\mathrm{OR}=1.072)$ were risk factors for MetS.

Figure 3 shows the nomogram of MetS risk prediction based on the LR model. First, the score points corresponding to each variable value were found, then all of the score points were summed to obtain the total score, and finally, the corresponding probability of MetS was determined. As an example, a person was 50 years old, the corresponding score was 43; no previous diabetes, the corresponding score was 41 ; no previous hypertension, the corresponding score was 41; fatty liver, the corresponding score was 59; no smoking, the corresponding score was 41 ; there was almost no exercise, the corresponding score was 41; the SBP was $129 \mathrm{mmHg}$, the corresponding score was 40; the DBP was $80 \mathrm{mmHg}$, the corresponding score was 38 ; the total score was 344 , so the probability of this person having MetS was 0.451 .

\section{Decision tree model}

The DT model, which had 7 depth layers and 18 leaves and was based on the random undersampling dataset, is shown in Fig. 4. BMI, as the root node of the DT, was the most important factor affecting MetS. The other variables are ranked in descending order of importance: SBP, diabetes, hypertension, DBP, fatty liver, smoking, and exercise.

\section{Discussion}

This study found that the prediction performance of the two classification models, LR and DT, was poor due to imbalanced datasets. However, the predictive performance of the classification model was significantly improved after balancing the dataset using random oversampling, SMOTE, random undersampling, and hybrid sampling, which indicated that resampling technology played an important role in improving the performance 


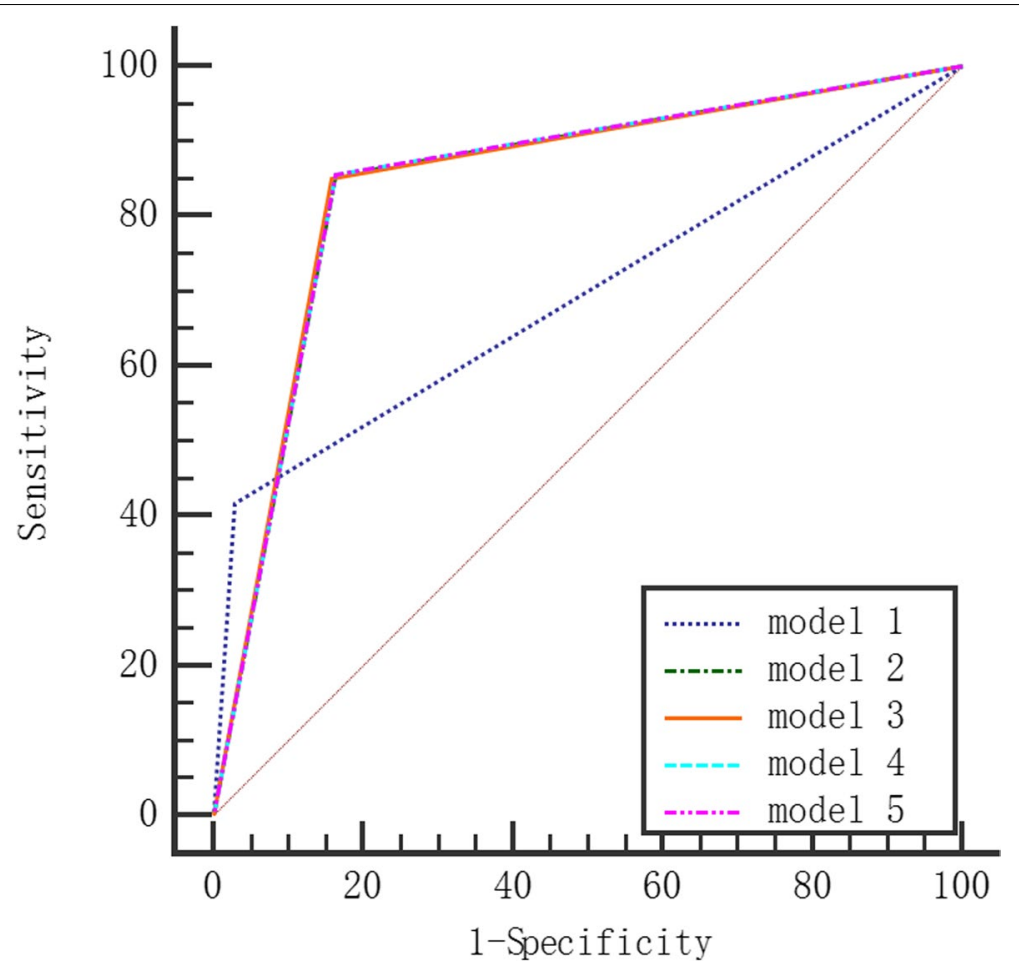

Fig. 1 ROC curve of LR model

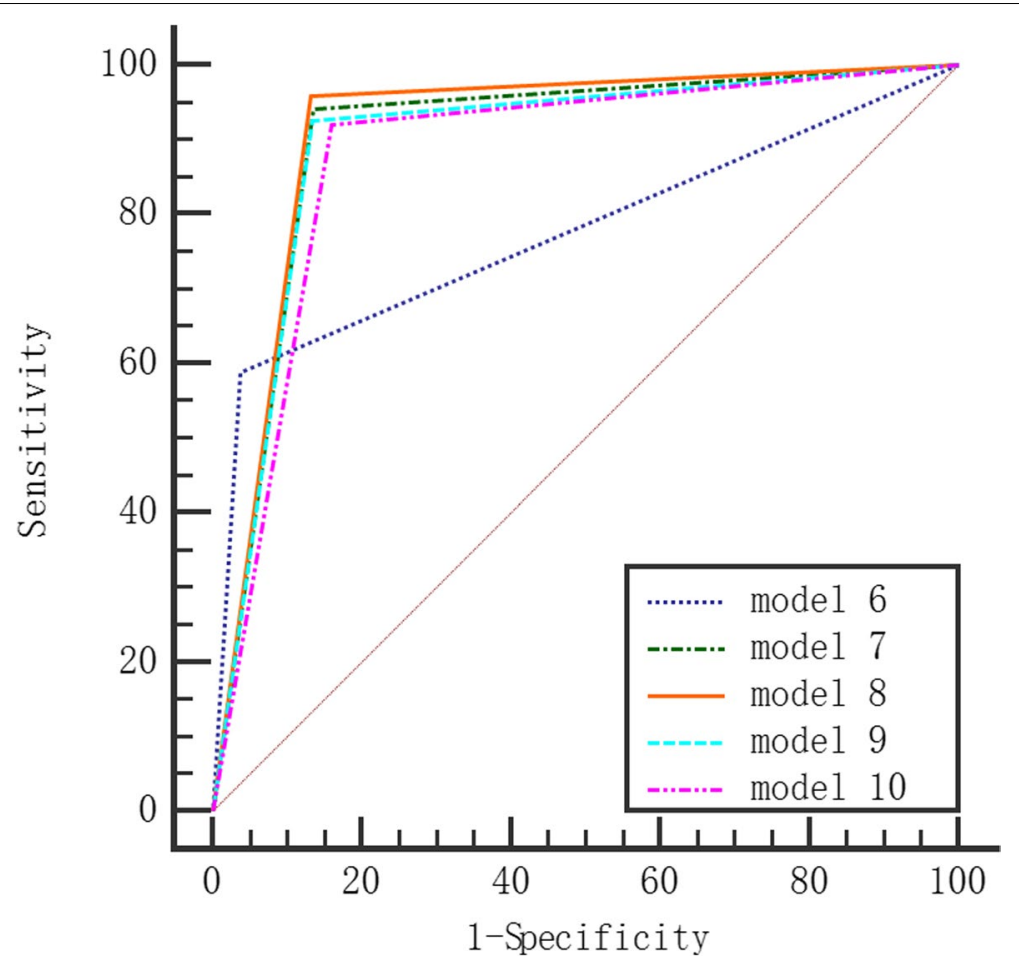

Fig. 2 ROC curve of DT model 
Table 3 Logistic regression analysis of influencing factors of MetS

\begin{tabular}{|c|c|c|c|c|c|c|}
\hline Variable & Coefficients & Std Error & Wald & OR & $95 \% \mathrm{Cl}$ & $P$ value \\
\hline Intercept & -12.744 & 0.437 & -29.152 & - & - & - \\
\hline \multicolumn{7}{|c|}{ Age (reference: 18 44) } \\
\hline $45 \sim 59$ & 0.139 & 0.098 & 1.418 & 1.149 & $0.948 \sim 1.391$ & 0.156 \\
\hline $60 \sim 74$ & 0.328 & 0.141 & 2.320 & 1.388 & $1.052 \sim 1.833$ & 0.020 \\
\hline $75 \sim 89$ & -0.117 & 0.274 & -0.427 & 0.890 & $0.522 \sim 1.531$ & 0.669 \\
\hline \multicolumn{7}{|c|}{ Previous diabetes (reference: No) } \\
\hline Yes & 2.186 & 0.178 & 12.281 & 8.902 & $6.333 \sim 12.739$ & $<0.001$ \\
\hline \multicolumn{7}{|c|}{ Previous hypertension (reference: No) } \\
\hline Yes & 1.040 & 0.121 & 8.619 & 2.830 & $2.238 \sim 3.593$ & $<0.001$ \\
\hline \multicolumn{7}{|c|}{ Fatty liver (reference: No) } \\
\hline Yes & 1.196 & 0.085 & 14.053 & 3.306 & $2.800 \sim 3.908$ & $<0.001$ \\
\hline \multicolumn{7}{|c|}{ Smoking (reference: No) } \\
\hline Quit & 0.325 & 0.169 & 1.925 & 1.384 & $0.996 \sim 1.930$ & 0.054 \\
\hline Yes & 0.432 & 0.091 & 4.731 & 1.541 & $1.288 \sim 1.844$ & $<0.001$ \\
\hline \multicolumn{7}{|c|}{ Exercise (reference: Hardly) } \\
\hline Run and others & -0.306 & 0.125 & -2.451 & 0.736 & $0.576 \sim 0.940$ & 0.014 \\
\hline Walk & -0.262 & 0.096 & -2.734 & 0.769 & $0.637 \sim 0.928$ & 0.006 \\
\hline SBP & 0.044 & 0.004 & 11.605 & 1.044 & $1.037 \sim 1.052$ & $<0.001$ \\
\hline DBP & 0.070 & 0.006 & 11.497 & 1.072 & $1.060 \sim 1.085$ & $<0.001$ \\
\hline
\end{tabular}

of the models on the imbalanced datasets. Among the four resampling methods, random undersampling had the best performance, which was different from the viewpoints of some previous studies [29-31]. Related studies found that random oversampling and SMOTE had better performances. We believe a possible reason is that the characteristics of the study data satisfied the conditions for the applicability of random undersampling. Random undersampling is suitable for a case where the number of samples of the majority class is very large, and the dataset is balanced by reducing the size of the redundant classes. In our study, 22,275 MetS subjects belonged to the majority class, and the larger sample size satisfied the application conditions of the random undersampling method. Therefore, random undersampling was the best method for balancing datasets in our research.

The two MetS risk prediction models constructed in this study, LR and DT, both had better prediction performance with AUROC values of 0.846 and 0.913 , respectively. However, for applications, not only the prediction performance of the risk prediction model but also the interpretability of the model is of concern. Interpretation of risk prediction models, even the simplest ones, is not straightforward for clinicians and their patients [32]. Previous studies have shown that patients prefer graphical representations of risks over digital risk estimates because this can improve their understanding of risks [33]. In this study, two visualization methods of the nomogram and tree diagrams of DT were used to show the risk of MetS occurrence, which are convenient for understanding the model results. We found that the results of the LR and DT models were basically consistent, both indicating that previous diabetes, previous hypertension, fatty liver, smoking, exercise, SBP and DBP are risk predictors for MetS. Notably, BMI, hypertension, diabetes, blood pressure, glucose, and lipid levels are already conventionally used in the diagnosis of MetS, and all have been shown to be risk factors for MetS. However, there were some differences in the results of the two models. The DT model showed that BMI was a very important risk predictor for MetS. Similarly, Jeong H S et al. also found that BMI was an important predictor of MetS [34]. In addition, the LR model showed that age was one of the risk predictors of MetS, and the risk of MetS in subjects aged $60 \sim 74$ years old was 1.388 times that of $18 \sim 44$ years old, which was similar to the findings of Wang $\mathrm{S}$ et al. [35]. Since both age and BMI are widely considered to be risk factors for MetS, if we only focus on the results of one model, it will lead to ignoring another important factor, so by combining LR and DT models, we can better identify risk factors for MetS.

The study results showed that DT had better predictive performance than LR on the original unbalanced and the balanced datasets. Thus, DT had a better prediction performance and ability to handle unbalanced data, which is similar to the research results of Sankari E S et al. [36]. DT is one of the interpretable models in 

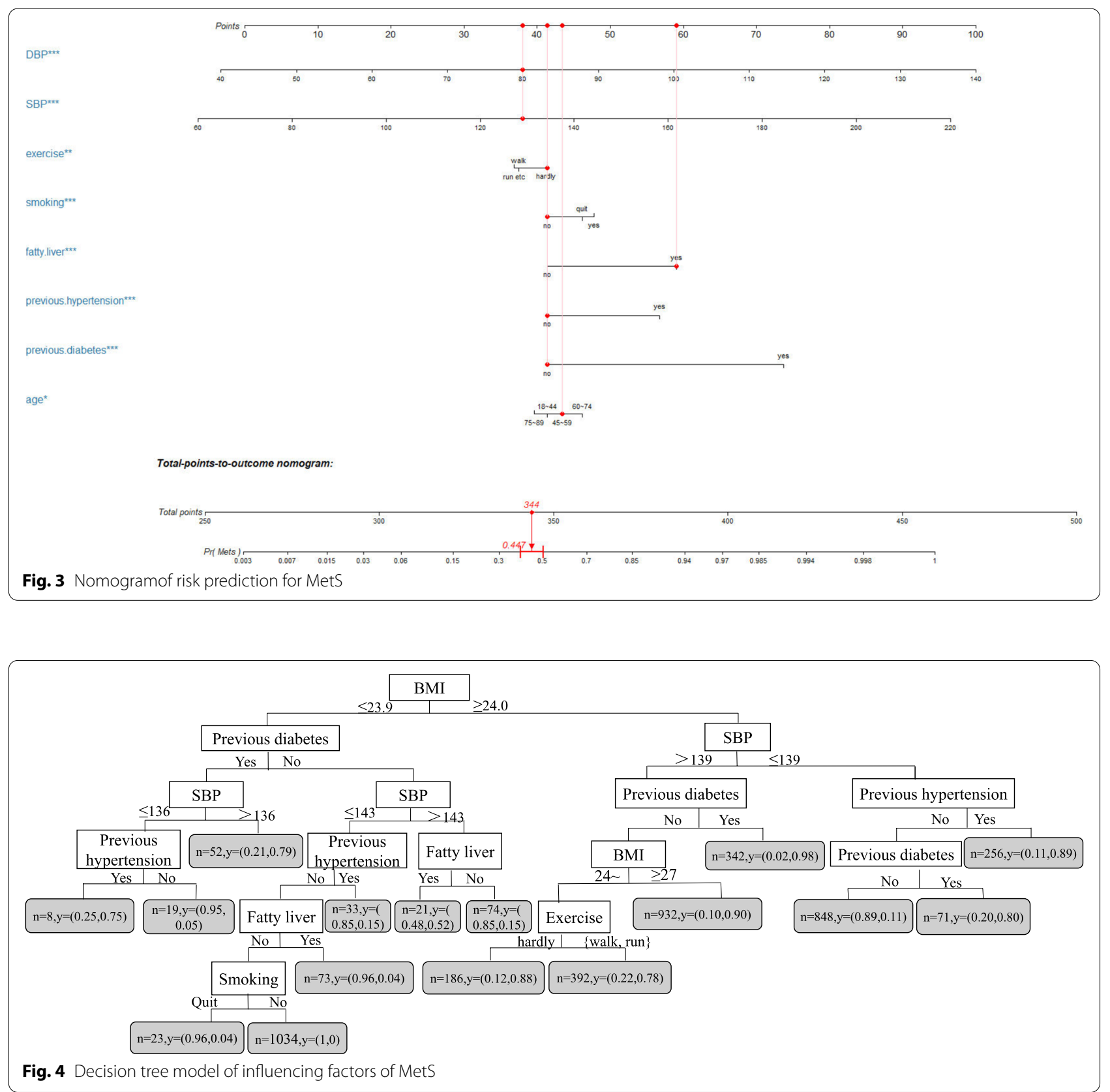

machine learning that can lead to a simple, clear, and intuitive tree structure to show the meaningful classification of prediction variables [37], eliminate the impact of collinearity between variables, and visually show the interactions between variables. In addition, it is of interest to us that the tree model can be used to generate easy-to-understand rules to guide the prevention and control of MetS. Currently, DT models have been applied in clinical practice, including disease diagnosis, risk of disease occurrence, regression and prognosis [38-40], health economics evaluation [41], clinical decision support system (CDSS) [42], and rational and safe drug use [43], and DT has shown powerful performance and high accuracy in all these aspects. Although studies have shown that the prediction performance of LR is lower than that of the DT, LR is not prone to overfitting and has good generalization ability [44].

Therefore, the MetS risk prediction model based on LR has a certain population promotion ability. In addition, since the LR model includes eight easily available indicators of age, SBP, DBP, exercise, smoking, fatty liver, hypertension and diabetes, the nomogram we 
developed on this basis has the advantages of being simple, intuitive and practical, can be used as a scoring tool for predicting the risk of MetS, and has some auxiliary value in clinical applications.

This study used the data preprocessing method resampling technique to deal with the data class imbalance problem. This study not only constructed the MetS risk prediction model based on two interpretable models, LR and DT, both with good prediction performance, but also used two visualization methods, nomograms and tree diagrams of DT, to present the model results. However, the MetS risk prediction models were developed and internally validated based on a physical examination population and we did not conduct external validation of the models. In addition, because this was a cross-sectional study, any associations observed in this study do not imply causal relationships.

\section{Conclusions}

The two interpretable models, DT and LR, have their own characteristics, and their combined use is complementary. Combining the two methods to construct an interpretable risk prediction model of MetS can provide methodological references for the prevention and control of MetS.

\begin{abstract}
Abbreviations
MetS: metabolic syndrome; non-MetS: nonmetabolic syndrome; BMI: body mass index; DBP: diastolic blood pressure; SBP: systolic blood pressure; OR: odds ratio; $\mathrm{Cl}$ : confidential interval; AUC: area under the curve; CHD: coronary heart disease; DT: decision tree; LR: logistic regression; AUROC: area under the receiver operating characteristic curve; ROC curve: receiver operating characteristic curve.
\end{abstract}

\section{Acknowledgements}

Not applicable.

\section{Authors' contributions}

Yan Zhang contributed to the study design, performed the statistical analyses, interpreted the data and drafted the manuscript. JAINA Razbek, Deyang Li, Lei Yang, Liangliang Bao, Wenjun Xia, Hongkai Mao, Xiaoxu Zhang and Mayisha Daken contributed to the statistical analyses and interpretation of the data and critically revised the manuscript. Mingqin Cao designed and supervised the study, interpreted the data, and critically revised the manuscript. All authors read and approved the final manuscript.

\section{Funding}

This research was supported by the National Natural Science Foundation of China with a grant (project number: 71663053).

\section{Availability of data and materials}

The datasets used and analysed during the current study are not publicly available due to the need to protect the individual privacy of the patients included in this study, but they are available from the corresponding author upon reasonable request.

\section{Declarations}

Ethics approval and consent to participate

This study was approved by the Ethics Committee of the First Affiliated Hospital of Xinjiang Medical University, and all methods were carried out in accordance with the relevant guidelines and regulations. The Ethics Committee of the First Affiliated Hospital of Xinjiang Medical University waived the need for informed consent.

\section{Consent for publication}

Not applicable.

\section{Competing interests}

The authors declare that they have no competing interests.

\section{Author details}

${ }^{1}$ Department of Epidemiology and Health Statistics, College of Public Health, Xinjiang Medical University, Urumqi, Xinjiang, China. ${ }^{2}$ Xinjiang De Kang Ci Hui Health Services Group, Urumqi, Xinjiang, China.

Received: 8 June 2021 Accepted: 17 January 2022

Published online: 08 February 2022

\section{References}

1. Tang Y, Zhao T, Huang N, Lin W, Luo Z, Ling C. Identification of Traditional Chinese Medicine Constitutions and Physiological Indexes Risk Factors in Metabolic Syndrome: A Data Mining Approach. Evidence-based complementary and alternative medicine. 2019;2019:1-10.

2. Federation I D. International Diabetes Federation (IDF) (2017) IDF Diabetes Atlas[EB/OL]. http://www.diabetesatlas.org/resources/2017-atlas.html.

3. Li R, Li W, Lun Z, Zhang H, Sun Z, Kanu J, et al. Prevalence of metabolic syndrome in Mainland China: a meta-analysis of published studies. BMC public health. 2016; 16:296.

4. Li R, Zhang L, Luo H, Lei Y, Zeng L, Zhu J, et al. Subclinical hypothyroidism and anxiety may contribute to metabolic syndrome in Sichuan of China: a hospital-based population study. Scientific reports. 2020; 10(1):2261.

5. Wu L, Shen Y, Hu L, Zhang M, Lai X. Prevalence and associated factors of metabolic syndrome in adults: a population-based epidemiological survey in Jiangxi province, China. BMC public health. 2020; 20(1):133.

6. Qin X, Qiu L, Tang G, Tsoi M, Xu T, Zhang L, et al. Prevalence of metabolic syndrome among ethnic groups in China. BMC public health. 2020; 20(1):297.

7. Liu L, Liu Y, Sun X, Yin Z, Li H, Deng K, et al. Identification of an obesity index for predicting metabolic syndrome by gender: the rural Chinese cohort study. BMC endocrine disorders. 2018; 18(1):54.

8. Ibrahim M, Pang D, Randhawa G, Pappas Y. Risk models and scores for metabolic syndrome: systematic review protocol. BMJ open. 2019; 9(9):e027326.

9. Li Y, Zhao L, Yu D, Wang Z, Ding G. Metabolic syndrome prevalence and its risk factors among adults in China: A nationally representative crosssectional study. PloS one. 2018; 13(6):e0199293.

10. Kong S, Cho Y. Identification of female-specific genetic variants for metabolic syndrome and its component traits to improve the prediction of metabolic syndrome in females. BMC medical genetics. 2019; 20(1):99.

11. Abd El-Wahab E, Shatat H, Charl F. Adapting a Prediction Rule for Metabolic Syndrome Risk Assessment Suitable for Developing Countries. Journal of primary care \& community health. 2019; 10:2150132719882760.

12. Talaei-Khoei A, Wilson J. Identifying people at risk of developing type 2 diabetes: A comparison of predictive analytics techniques and predictor variables. International journal of medical informatics. 2018; 119:22-38.

13. O'Neill A, Yang D, Roy M, Sebastiampillai S, Hofer S, Xu W. Development and Evaluation of a Machine Learning Prediction Model for Flap Failure in Microvascular Breast Reconstruction. Annals of surgical oncology. 2020; 27(9):3466-3475.

14. Geldof T, Van Damme N, Huys I, Van Dyck W. Patient-Level Effectiveness Prediction Modeling for Glioblastoma Using Classification Trees. Frontiers in pharmacology. 2019; 10:1665. 
15. Elshawi R, Al-Mallah M, Sakr S. On the interpretability of machine learning-based model for predicting hypertension. BMC Med Inform Decis Mak. 2019; 19(1):146.

16. Lu YH, Lu JM, Wang SY, Li CL, Pan CY. Comparison of the diagnostic criteria of metabolic syndrome by International Diabetes Federation and that by Chinese Medical Association Diabetes Branch. Zhonghua yi xue za zhi. 2006; 86(6):386-389.

17. Schomaker M, Heumann C. Bootstrap inference when using multiple imputation. Statistics in medicine. 2018; 37(14):2252-2266.

18. Boussat B, François O, Viotti J, Seigneurin A, Giai J, François P, et al. Managing Missing Data in the Hospital Survey on Patient Safety Culture: A Simulation Study. J Patient Safety. 2021;17(2):e98-106.

19. Chen J, Lalor J, Liu W, Druhl E, Granillo E, Vimalananda V, et al. Detecting Hypoglycemia Incidents Reported in Patients'Secure Messages: Using Cost-Sensitive Learning and Oversampling to Reduce Data Imbalance. J Med Internet Res. 2019; 21(3):e11990.

20. Xie C, Du R, Ho JW, Pang HH, Chiu KW, Lee EY, et al. Effect of machine learning re-sampling techniques for imbalanced datasets in 18F-FDG PET-based radiomics model on prognostication performance in cohorts of head and neck cancer patients. Eur J Nuclear Med Mol Imaging. 2020;47(12):2826-35.

21. Feng $X$, Yang $L$, Tan L, Li Y. Risk factor analysis of device-related infections: value of re-sampling method on the real-world imbalanced dataset. BMC Med Inform Decis Mak. 2019; 19(1):185.

22. Fotouhi S, Asadi S, Kattan M. A comprehensive data level analysis for cancer diagnosis on imbalanced data. J Biomed Informatics. 2019;90:103089.

23. Chawla NV, Bowyer KW, Hall LO, Kegelmeyer WP. SMOTE: Synthetic Minority Over-sampling Technique. 2011.

24. Wang Y, Du Z, Lawrence W, Huang Y, Deng Y, Hao Y. Predicting Hepatitis B Virus Infection Based on Health Examination Data of Community Population. Int J Environ Res Public Health. 2019;16(23):4842.

25. Zhang J, Li X, Huang R, Feng W, Kong Y, Xu F, et al: A nomogram to predict the probability of axillary lymph node metastasis in female patients with breast cancer in China: A nationwide, multicenter, 10-year epidemiological study. Oncotarget. 2017; 8(21):35311-35325.

26. Tayefi M, Esmaeili H, Saberi Karimian M, Amirabadi Zadeh A, Ebrahimi M, Safarian M, et al. The application of a decision tree to establish the parameters associated with hypertension. Computer Methods Programs Biomed. 2017;139:83-91.

27. Mayo M, Chepulis L, Paul R. Glycemic-aware metrics and oversampling techniques for predicting blood glucose levels using machine learning. PloS one. 2019; 14(12):e0225613.

28. Li B, Ding S, Song G, Li J, Zhang Q. Computer-Aided Diagnosis and Clinical Trials of Cardiovascular Diseases Based on Artificial Intelligence Technologies for Risk-Early Warning Model. J Med Systems. 2019;43(7):228.

29. Wu Y, Fang Y. Stroke Prediction with Machine Learning Methods among Older Chinese. Int J Environ Res Public Health. 2020;17(6):1828.

30. Lanera C, Berchialla P, Sharma A, Minto C, Gregori D, Baldi I. Screening PubMed abstracts: is class imbalance always a challenge to machine learning? Systematic reviews. 2019; 8(1):317.

31. Blagus R, Lusa L. Joint use of over- and under-sampling techniques and cross-validation for the development and assessment of prediction models. BMC Bioinformatics. 2015;16:363.

32. Van Belle V, Van Calster B. Visualizing Risk Prediction Models. PloS one. 2015; 10(7):e0132614.

33. Lipkus IM, Hollands JG. The Visual Communication of Risk. Journal of the National Cancer Institute Monographs. 1999; 25(25):149.

34. Jeong $\mathrm{H}$. The Relationship between Workplace Environment and Metabolic Syndrome. Int J Occupational Environ Med. 2018;9(4):176-83.

35. Wang S, Wang S, Jiang S, Ye Q. An anthropometry-based nomogram for predicting metabolic syndrome in the working population. Eur J Cardiovasc Nurs. 2020;19(3):223-9.

36. Sankari E, Manimegalai D. Predicting membrane protein types by incorporating a novel feature set into Chou's general PseAAC. J Theoretical Biol. 2018;455:319-28.

37. Deng X, Yu T, Hu A. Predicting the Risk for Hospital-Acquired Pressure Ulcers in Critical Care Patients. Critical Care Nurse. 2017;37(4):e1-11.

38. Speiser J, Callahan K, Houston D, Fanning J, Gill T, Guralnik J, et al. Machine Learning in Aging: An Example of Developing Prediction Models for Serious Fall Injury in Older Adults. J Gerontol Series. A Biol Sci Med Sci. 2021;76(4):647-54.
39. Madakkatel I, Zhou A, McDonnell M, Hyppönen E. Combining machine learning and conventional statistical approaches for risk factor discovery in a large cohort study. Scientific Reports. 2021;11(1):22997.

40. Ghazalbash S, Zargoush M, Mowbray F, Papaioannou A. Examining the predictability and prognostication of multimorbidity among older Delayed-Discharge Patients: A Machine learning analytics. Int J Med Informatics. 2021;156:104597.

41. McManus E, Sach T, Levell N. An introduction to the methods of decisionanalytic modelling used in economic evaluations for Dermatologists. J Eur Acad Dermatol Venereol. 2019;33(10):1829-36.

42. Yu HW, Hussain M, Afzal M, Ali T, Choi JY, Han HS, et al. Use of mind maps and iterative decision trees to develop a guideline-based clinical decision support system for routine surgical practice: case study in thyroid nodules. J Am Med Inform Assoc. 2019;26(6):524-36.

43. Chen SS, Zaborek NA, Doubleday AR, Schaefer SC, Long KL, Pitt SC, et al. Optimizing Levothyroxine Dose Adjustment After Thyroidectomy With a Decision Tree. J Surg Res. 2019;244:102-6.

44. Guo HP, Dong YD, Wu CA, Fan M. Logistic regression method for class imbalance problem. Moshi Shibie yu Rengong Zhineng/Pattern Recognition and Artificial Intelligence. 2015; 28(8):686-693.

\section{Publisher's Note}

Springer Nature remains neutral with regard to jurisdictional claims in published maps and institutional affiliations.

Ready to submit your research? Choose BMC and benefit from

- fast, convenient online submission

- thorough peer review by experienced researchers in your field

- rapid publication on acceptance

- support for research data, including large and complex data types

- gold Open Access which fosters wider collaboration and increased citations

- maximum visibility for your research: over $100 \mathrm{M}$ website views per year

At BMC, research is always in progress.

Learn more biomedcentral.com/submissions 